\title{
MULTIPORT MICRO-OPTIC DEVICES FOR HOLLOW CORE FIBRE APPLICATIONS
}

\author{
Yongmin Jung*, Thomas D. Bradley, John R. Hayes, Gregory Jasion, Ian A. Davidson, \\ Hesham Sakr, Yong Chen, Francesco Poletti, David J. Richardson
}

\author{
Optoelectronics Research Centre, University of Southampton, Southampton, SO17 1BJ, UK, \\ *ymj@orc.soton.ac.uk
}

\author{
Keywords: HOLLOW CORE FIBRES, MICROSTRUCTURED OPTICAL FIBRES, OPTICAL FIBRE \\ COMPONENTS
}

\begin{abstract} a broader range of fibre optic applications.

\section{Introduction}

Hollow core fibres (HCFs) based on photonic bandgap or antiresonant effects [1] guide light in a hollow air containing region rather than a conventional solid glass core. As a result HCFs present very attractive optical properties such as low latency (and/or low nonlinearity) data transmission for telecommunications, high damage and nonlinear thresholds for high power (or high energy) laser beam delivery, and low Rayleigh scattering and good thermal stability for high sensitivity gyroscope/sensor applications [2-3]. While such fibres have been available for several years, there is still a lack of reliable HCF components, and only a few fibre optic devices (the majority for fibre termination and inter-connection) are commercially available. Due to this, solid-core fibre optic components (e.g. couplers, isolators, WDM filters etc.) are the only possible solution in many HCF applications, requiring solid to HCF splicing which generally degrades performance (e.g. through increased losses, due to splicing, and back reflections, due to the $4 \%$ Fresnel reflection from flat air/glass interfaces). Moreover, this often necessitates the use of very short pigtail lengths to minimize the impact of the solid-core fibres properties themselves.
\end{abstract}

Using micro-optic collimator technology, we present fully integrated 3-port hollow-core-fibre (HCF) components (e.g. 1×2 beam-splitter and WDM coupler), thereby increasing the diversity of inline HCF components and facilitating the use of HCF in

Very recently, using micro-optic collimator technology [4], we have presented a compact air gap device for HCFs that can be used to construct various 2-port HCF devices (e.g. isolators, bandpass filters) with excellent optical performance [5]. In this paper, we further extend our work to multiport $\mathrm{HCF}$ components and two exemplary 3-port HCF devices are fabricated with reasonable excess loss $(\sim 2 \mathrm{~dB})$, good modal purity $(>20 \mathrm{~dB})$ and low Fresnel back reflections $(<-45 \mathrm{~dB})$.

Fig. 1 shows a schematic of an exemplar 3-port HCF component based on our micro-optic collimator assembly, which comprises a dual fibre collimator (left), a single fibre collimator (right) and a functional element in the middle. To prove the concept, we use a 50/50 dielectric beam splitter chip (size $=1.4 \mathrm{~mm} \times 1.4 \mathrm{~mm}$ ) to make a $3 \mathrm{~dB}$ beam splitter, and a dichroic mirror to make a WDM coupler. In order to make these 3-port HCF devices, we first fabricated a dual fibre collimator using HCFs (left assembly in Fig. 1). Two flatcleaved HCFs were inserted into a dual-hole glass ferrule (inner dia. $=500 \mu \mathrm{m}$, outer dia. $=1.8 \mathrm{~mm}$ ) and placed at the focal point of a short graded-index (GRIN) lens (pitch distance $=0.25$ effective focal length $=1.99 \mathrm{~mm}$ ) to create two collimated beams. Next, a 50/50 beam splitter chip was placed on a highprecision flexure stage and actively aligned to a high degree of accuracy by monitoring the reflected power (Port $1 \rightarrow$ Port 2 ). We used a GRIN lens with a flat surface in the dual fibre

\section{2. $1 \times 2$ beam splitter for HCFs}

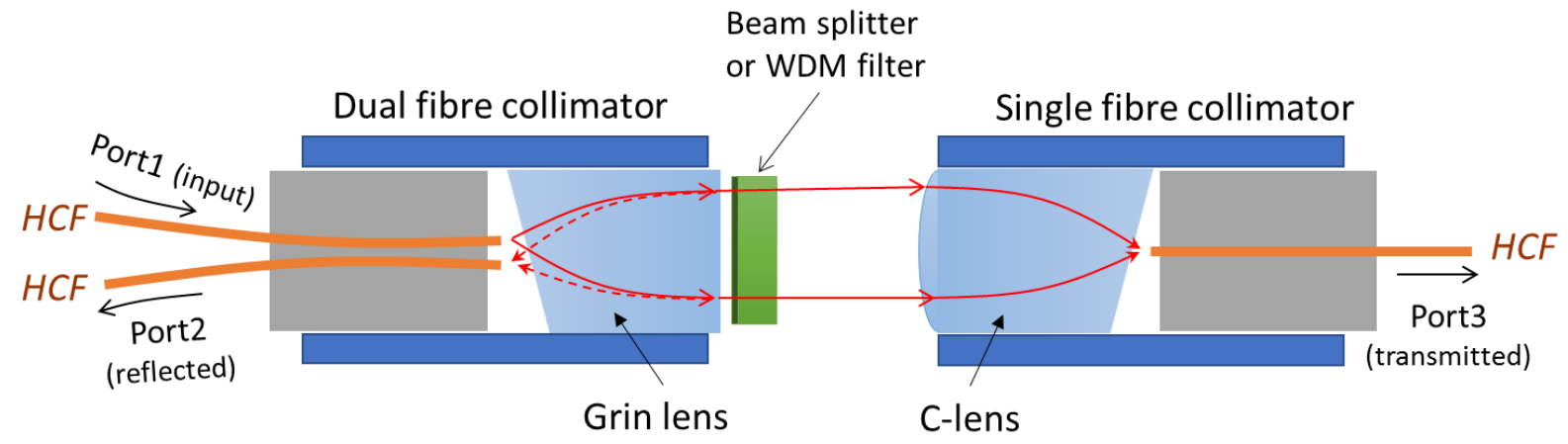

Fig.1 A schematic of 3-port hollow core fibre components based on micro-optic collimator assembly. 
collimator to allow ready placement and final attachment of the mirror. In a second step, we assembled a single HCF collimator (right assembly in Fig. 1), to produce a well collimated beam, this time using a C-lens (focal length $=2.98$ $\mathrm{mm}$ ). Finally, these two collimator assemblies were combined together to realize our 3-port HCF device. Again precise active alignment was used to guarantee low loss interconnection from port 1 to port 3 .

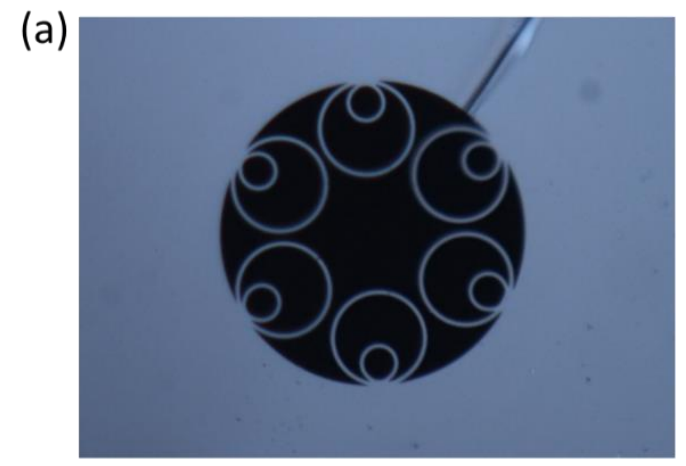

(b)

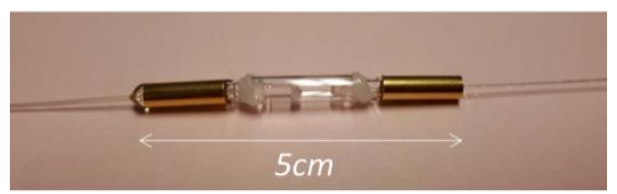

Fig.2 (a) Microscope image of HCF cross-section and (b) the assembled 3-port HCF device.

A nested anti-resonant nodeless fibre (NANF) [6] was used as an exemplar $\mathrm{HCF}$ in our experiment. A cross-sectional microscope image of the fibre is shown in Fig. 2(a). Six double nested capillaries form an anti-resonant cladding to provide efficient, low loss light guidance in the second anti-resonance window. The fibre has a core diameter of $34 \mu \mathrm{m}$, a microstructured cladding diameter of $90.6 \mu \mathrm{m}$ and an outer diameter of $238 \mu \mathrm{m}$. The fibre loss was $\sim 2.8 \mathrm{~dB} / \mathrm{km}$ at $1550 \mathrm{~nm}$ and the fibre had a $>300 \mathrm{~nm}$ transmission bandwidth. Note that this particular fibre supports single mode guidance, however if higher-order modes are excited at the input (particularly the $\mathrm{LP}_{11}$ mode) they can survive to a detectable level over short fibre lengths (e.g. a few meter to a few tens of metres) despite the relatively high differential modal loss of these fibres. We have examined the modal properties of this fibre in some detail, as will be discussed later when describing the performance of the fabricated HCF devices. Fig. 2(b) shows a photograph of a fully-assembled 3-port HCF prototype device. The two fibre collimators and associated functional element are hermetically-sealed inside a protective glass tube and a further metal tube and/or fibre optic connector boots can be added for increased mechanical protection. The total device length was $\sim 5 \mathrm{~cm}$.

Fig. 3 shows the reflection and transmission spectra of the fabricated $(1 \times 2)$ HCF-integrated beam splitter, which we measured using a superluminescent diode and an optical spectrum analyser. The power-splitting ratio is relatively equal from $1500 \mathrm{~nm}$ to $1620 \mathrm{~nm}$ and the excess loss was measured to be $\sim 2 \mathrm{~dB}$. Here, the particular split-ratio is defined by the beam splitter chip used in our experiment and any desired- split-ratio could be readily achieved through a different choice of dielectric beam splitter.

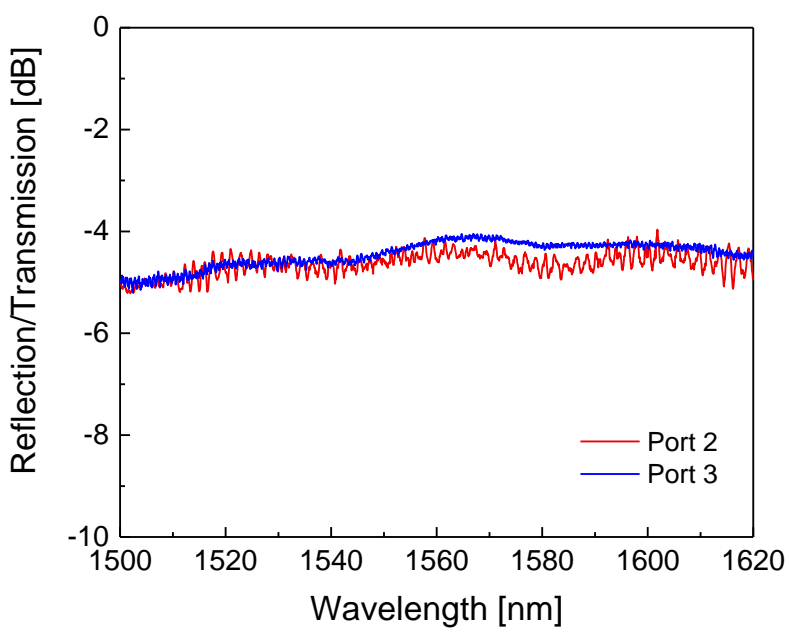

Fig. 3 Reflection and transmission spectra of the fabricated HCF (1×2) beam splitter.

For the reflection port (Port 2), a slight oscillatory spectral response is observed. We attribute this to intermodal interference resulting from the excitation of a small higher order mode (HOM) component at the reflection port originating from imperfection in the fibre collimator alignment coupled with the use of a short (few metre) HCF pigtail that was insufficient to suppress all HOM content. We note that these HOMs can be eliminated either through the use of a longer fibre length (benefiting from the substantially higher loss of HOMs versus the fundamental), or if the short tails are tightly looped or bent (exploiting the increased bend loss sensitivity of HOMs).

(a)

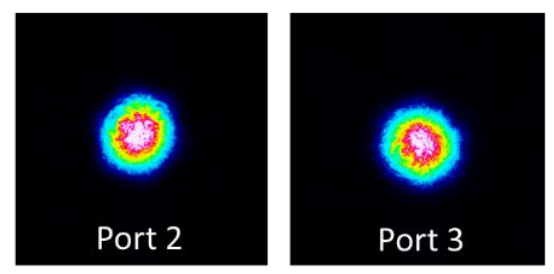

(b)

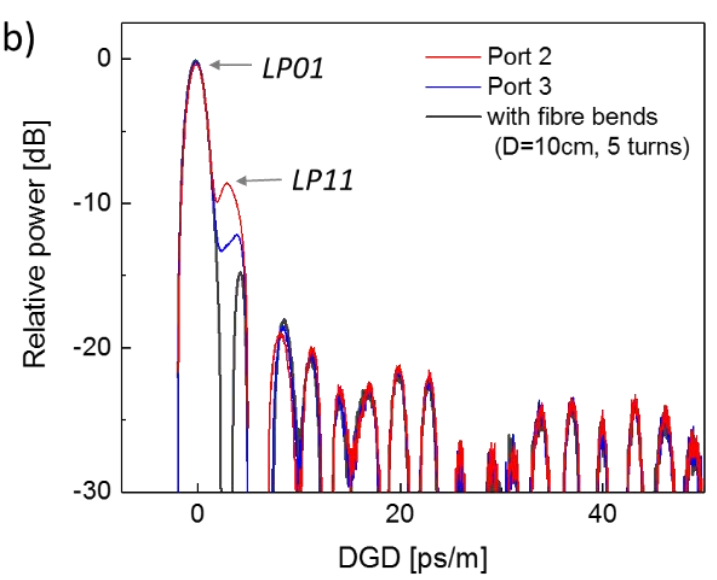

Fig.4 (a) Beam profiles from two output ports and (b) the impulse response of the device after propagating a $30 \mathrm{~m}$ of HCF. 
In order to visually assess the beam quality of the fabricated HCF $(1 \times 2)$ beam splitter, both output ports were first examined using a CCD camera. As shown in Fig. 4(a), quite clean fundamental mode profiles were observed at both fibre ports. However, note that these CCD beam profiling can be used only for quick identification of the dominant spatial modes, not quantitative modal analysis. Therefore, we further analyzed the modal quality of the device with a time-of-flight measurement by splicing an additional $30 \mathrm{~m}$ of HCF, which enables enough intermodal delay to be discernible in the time domain. As shown in Fig. 4(b), a small $\mathrm{LP}_{11}$ peak can be identified at a differential group delay (DGD) of $2.6 \mathrm{ps} / \mathrm{m}$ and modal purity of the outputs (i.e. $\mathrm{LP}_{01}$ intensity compared to other HOMs) was estimated to be $\sim 8.4 \mathrm{~dB}$ for port $2(\sim 12 \mathrm{~dB}$ for port 3), respectively. Note that the modal purity of the device highly depends on the fibre alignment during the assembly and the center-to-center fibre alignment is critical to minimize this inter-modal crosstalk. However, as mentioned before, these HOMs can be effectively suppressed by bending the fibre without significantly affecting the $\mathrm{LP}_{01}$ mode loss and a high purity $\mathrm{LP}_{01}$ mode ( $>20 \mathrm{~dB}$ extinction ratio) is readily achieved with optimum fibre bending (e.g. $\mathrm{D}=10 \mathrm{~cm}, 5$ turns) as shown in the gray colored trace in Fig. 4(b). Here, the LP 11 modal peak at $\mathrm{DGD}=2.6 \mathrm{ps} / \mathrm{m}$ is seen to be completely eliminated and only detector ringing artifacts associated with the impulse response of the detector can be observed. Moreover, this HOM suppression can also be realized by employing a longer length of fibre and we have confirmed this by splicing an extra $160 \mathrm{~m}$ length of HCF on a fibre bobbin $(\mathrm{D}=30 \mathrm{~cm})$ to output 2 and observing that $>20 \mathrm{~dB}$ modal purity can be readily achieved. We also measured the back reflection of the fabricated device and confirmed that it remains very low $(<-45 \mathrm{~dB})$ because there is no flat glass/air interface within the full optical path. This ensures that such HCF devices are compatible with many high sensitivity sensor, spectroscopy and gyroscope applications.

\section{WDM coupler for HCFs}

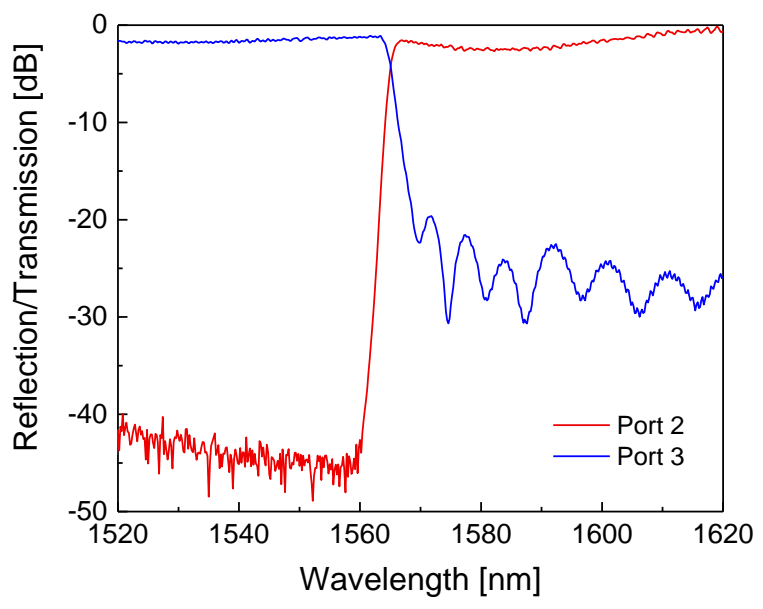

Fig.5 Reflection and transmission spectra of WDM coupler for HCFs

As another example of a 3-port HCF device, we have fabricated a wavelength division multiplexing (WDM) coupler using a dichroic mirror chip (e.g. a C/L-band splitter in our demonstration) placed between two HCF collimators. This chip is commercially designed to be used for the splitting of telecom C-band (reflection from 1500 to $1564 \mathrm{~nm}$ ) and L-band (transmission from 1570 to $1620 \mathrm{~nm}$ ) channels. This WDM functionality is readily integrated into $\mathrm{HCF}$ devices as shown in Fig. 5. The insertion loss of the HCF-WDM coupler was $~ 2$ $\mathrm{dB}$ at both $\mathrm{C}$ - and L-bands (including the insertion loss of the dichroic mirror $(\sim 0.6 \mathrm{~dB}))$. We can see a small signature of sinusoidal spectral fluctuation in the L-band, which is mainly due to thin-film interference effects associated with the dichroic mirror used. Note that such HCF-WDM couplers should prove very useful in many laser and amplifier configurations (especially, gas or dye lasers in HCF), Raman spectroscopy and pump-probe applications using HCF.

\section{Conclusion}

We have incorporated hollow-core fibres in a micro-collimator assembly and successfully developed 3-port HCF components (e.g. exemplary $1 \times 2$ beam splitter and WDM coupler devices). Micro-optic collimator technology provides a robust, diverse and enabling platform to incorporate various optical functionalities into HCF systems and it can be further extended to more ports (e.g. 4-port or even more) for various $\mathrm{HCF}$ applications.

\section{Acknowledgements}

This work was supported in part by the EPSRC funded "Airguide Photonics" Programme Grant (EP/P030181/1), the EPSRC National Hub in High Value Photonic Manufacturing (EP/N00762X/1) and ERC Lightpipe project (682724).

\section{References}

\section{Journal articles}

[1] F. Poletti et al.: 'Toward high-capacity fibre-optic communications at the speed of light in vacuum,' Nat. Photonics, 2013, 7(4) 279-284.

[2] P. St. J. Russel et al.: 'Hollow-core photonic crystal fibres for gas-based nonlinear optics,' Nat. Photonics, 2014, 8, 278286.

[3] S. Blin et al.: 'Reduced thermal sensitivity of a fiber optic Gyroscope using an air-core photonic bandgap fiber,' J. Lightwave Technol., 2007, 25(3), 861-865.

[4] Y. Jung et al.: 'Fully integrated optical isolators for space division multiplexed (SDM) transmission,' APL Photonics., 2019, 4, 022801.

\section{Conference Paper}

[5] H. Kim et al.: 'Free space based hollow core fiber interconnection and associated in-line components,' in Proc. OFC, San Diego, USA, Mar 2019, Th3E.3.

[6] T. D. Bradley et al.: 'Record low-loss $1.3 \mathrm{~dB} / \mathrm{km}$ data transmitting antiresonant hollow core fibre,' in Proc. ECOC, Rome, Italy, Sep 2018, Th3F.2 